\title{
Clinical efficacy of intersphincteric resection for low rectal cancer compared with abdominoperineal resection: a single-center retrospective study
}

\section{Baifu Peng}

Guangzhou First People's Hospital

\section{Zixin Wu}

Guangzhou First People's Hospital

\section{Zijian He}

Guangzhou First People's Hospital

Guanwei Li

Guangzhou First People's Hospital

Jie Cao

Guangzhou First People's Hospital

Wanglin Li ( $\Delta$ 421255910@qq.com )

Guangzhou First People's Hospital

\section{Research}

Keywords: intersphincteric resection, abdominoperineal resection, low rectal cancer

Posted Date: May 13th, 2020

DOI: https://doi.org/10.21203/rs.3.rs-26040/v1

License: (c) (1) This work is licensed under a Creative Commons Attribution 4.0 International License. Read Full License 


\section{Abstract \\ Purpose}

In recent years, intersphincteric resection (ISR) has been increasingly used to replace abdominoperineal resection (APR) for low rectal cancer. This study was to compare the clinical efficacy of ISR and APR.

\section{Methods}

Between 2012 and 2018, 74 consecutive patients with low rectal cancer underwent ISR and APR in our medical centre. The outcomes were retrospectively studied and compared.

\section{Results}

A total of 43 patients underwent ISR and 31 underwent APR were included in the study. No significant differences were found between two groups in gender, age, BMI and ASA score. ISR group showed shorter operative time $(P=0.02)$ and less blood loss $(P=0.001)$. Hospital stays, time to soft diet, and postoperative thirty-day complications were not significantly different between the two groups. As for the long-term outcomes, the survival and recurrence rate were similar between two groups. Moreover, LARS score and Wexner score showed the anal function after ISR was generally satisfactory.

\section{Conclusion}

This study suggested that ISR may provide a feasible alternative to APR, with superior short-term outcomes, similar oncological outcomes and satisfactory postoperative anal function.

\section{Introduction}

According to the global tumor statistical analysis, the incidence of colorectal cancer has increased year by year in recent years, in both sexes combined, the incidence rate of colorectal cancer ranks fourth, and the mortality rate ranks second. The rectal cancer, especially the low rectal cancer, which is $5 \mathrm{~cm}$ or less from the anal verge is more common, accounting for $70-80 \%$ of the total number of rectal cancer, which may be related to dietary habits, social environment, genetics and other factors [1, 2]. At present, the treatment of low rectal cancer mainly adopts the comprehensive treatment with surgery as the main and chemoradiotherapy as the auxiliary. Radical treatment of tumor, as far as possible to retain the anal shape, anal sphincter integrity and urogenital function has become its main therapeutic purposes. Abdominoperineal resection (APR) is a standard operation for low rectal cancer, completely removes the distal colon, rectum, and anal sphincter complex to radically resect the tumor, resulting in a permanent colostomy ineluctably, which greatly influences the patient's quality of life [3]. In recent years, intersphincteric resection (ISR) has been performed at more and more specialized institutions to extend 
the opportunity for anus preservation for low rectal cancer [4]. However, there are also potential risks associated with ISR, including the risk of tumor recurrence and problems with the patient's anal function after surgery. To date, only a few studies have compared the long-term outcomes of ISR with APR and reported the postoperative anal function in patients after ISR.

In this study, the short-term and oncological outcomes, including disease-free survival and local recurrence, of ISR and APR for low rectal cancer in our medical centre were compared. Moreover, we conducted a preliminary follow-up and reported on the postoperative anal function of patients who underwent ISR.

\section{Methods}

\section{Patients}

From January 2012 to December 2018, a total of 464 patients with rectal cancer underwent curative surgical treatment in our medical center. Among them, 43 patients underwent ISR and 31 patients underwent APR who had invasive rectal cancer located within $5 \mathrm{~cm}$ from the anal verge were eligible and included in this study (Fig. 1). The study was approved by the Institutional Review Board of our hospital, and all patients provided written informed consent.

All patients underwent routinely assessed preoperatively with digital anorectal examination, colonoscopy, pelvic magnetic resonance imaging (MRI) and/or endorectal ultrasonography, thoracoabdominal computed tomography (CT) scan, and sphincter manometry. Inclusion criteria was histologically proven low rectal adenocarcinoma. Exclusion criteria included colon cancer or upper rectal cancer, tumor distant metastasis (clinical stage $\otimes$ period), emergent setting, such as acute bowel obstruction or perforation from cancer [5], inflammatory bowel disease and familial polyposis. Moreover, studies have shown that the onlogical outcomes of the patients accepted neoadjuvant chemoradiation could be improved [6, 7], so this part of patients were not be included in this study.

\section{Data Collection And Definitions}

Data on patients' characteristics and short-term outcomes were obtained from medical records and examination results. The basic diseases of patients in this study included hypertension, diabetes, heart disease and chronic bronchitis. Postoperative thirty-day complications were classified by Clavien-dindo scoring system [8]. Postoperative anal function was assessed among all patients who received closure of the ileostomy after ISR. The LARS score and Wexner score were used to assess the anal function and faecal continence $[9,10]$. LARS score includes 5 items: exhaust control disorder ( $0 \sim 7$ points), loose feces ( $0 \sim 3$ points), defecation frequency ( $0 \sim 5$ points), re-defecation within 1 hour after defecation $(0 \sim 11$ points), and defecation urgency ( $0 \sim 16$ points). According to the score ( $0 \sim 42$ points), it was divided into 3 degrees: 0 20 points indicates "no low anterior resection syndrome"; Scores ranging from 21 to 29 indicate "mild low preresection syndrome", while scores ranging from 30 to 42 indicate "severe low 
preresection syndrome". The Wexner score is a questionnaire that indicates the severity of fecal incontinence. It consists of 5 items, including the type and frequency of incontinence (solid, liquid, gas and whether to use pads) and lifestyle changes. The total score is 20 points. A score of $\geq 10$ is an indicator for stool incontinence. The higher the score, the worse the anal function. Patients were followed up at 6-month intervals for 2 years, and once annually thereafter. Follow-up examinations included physical examination, serum tumor marker assay, chest $\mathrm{X}$ ray or $\mathrm{CT}$, abdomino-pelvic $\mathrm{CT}$ or $\mathrm{MRI}$, and colonoscopy. Recurrence was determined by imageological examinations and/or pathological confirmation. Local recurrence was defined as recurrent disease in the pelvis, including the anastomosis. Distant metastasis was defined as recurrent disease outside the pelvis. Follow-up and anal function data were obtained by telephone interviews or outpatient reexamination.

\section{Statistical Analysis}

All statistical analyses were conducted using software SPSS 16.0. Quantitative data were reported as mean, median, standard deviation (SD), and interquartile range (25-75\%). Student's t-test and chisquared test (or Fisher's exact test) were used to compare normally distributed continuous variables and categorical variables, respectively. Survival curves were performed using the Kaplan-Meier method, and the difference between curves was assessed by the log-rank test. P values $<0.05$ was considered statistically significant.

\section{Results}

\section{Patient Characteristics}

Of the 74 patients who are included for analysis, 43 underwent ISR and 31 underwent APR in our center. There were no significant differences with regard to age, sex, BMI, ASA score, basic diseases and the previous abdominal surgery between the groups (Table 1). All patients in both groups had not received neoadjuvant chemoradiotherapy. 
Table 1

Patients' Features

\begin{tabular}{|lllr|}
\hline & $\begin{array}{l}\text { ISR(n=43)n(\%)or } \\
\text { mean[SD] }\end{array}$ & $\begin{array}{l}\text { APR(n=31) } n(\%) \text { or } \\
\text { mean[SD] }\end{array}$ & $P$ \\
\hline Sex (male) & $22(51.2)$ & $17(54.8)$ & 0.755 \\
\hline Age (years) & $64.7[11.1]$ & $65.2[9.7]$ & 0.837 \\
\hline Body mass index $(\mathrm{kg} / \mathrm{m} 2)$ & $22.4[2.8]$ & $22.3[2.4]$ & 0.849 \\
\hline ASA score & & & 0.996 \\
\hline 1 & $15(34.9)$ & $11(35.5)$ & \\
\hline 2 & $24(55.9)$ & $17(54.5)$ & 0.579 \\
\hline 3 & $4(9.2)$ & $3(10)$ & 0.658 \\
\hline Basic diseases(+) & $18(41.90)$ & $11(35.5)$ & \\
\hline $\begin{array}{l}\text { Previous abdominal surgery } \\
\text { history(+) }\end{array}$ & $4(9.3)$ & $2(6.5)$ & \\
\hline ASA, American Society of Anesthesiologists; SD, standard deviation. & \\
\hline
\end{tabular}

\section{Short-term Outcomes}

Operative time (mean 261.8 min ISR; 319.2 min APR; P = 0.021) and blood loss (mean $185.8 \mathrm{~mL}$ ISR; $375.0 \mathrm{~mL}$ APR; $P=0.001$ ) were significantly lower in the ISR group compared with the APR group. The rate of 30-day postoperative complications (20.9\% versus $25.8 \%$ ) and Clavien-Dindo score were similar between the ISR and APR groups. In the ISR group, postoperative complications occured in 9 patients, including 3 patients with anastomotic leakage, which was the most common complication in the ISR group. In the APR group, the most common complication was hemorrhage. One patient in the ISR group died in the hospital due to pulmonary infection, while there was no in-hospital mortality in APR group. The ISR group showed no difference from the APR group in time to anal or stoma exhaust $(P=0.953)$, time to soft diet $(P=0.248)$, postoperative hospital stay $(P=0.087)$ and thirty-day reoperation or readmission $(P$ $=0.814)($ Table 2$)$. 
Table 2

Short-Term Outcomes

\begin{tabular}{|c|c|c|c|}
\hline & $\begin{array}{l}\text { ISR }(n=43) n(\%) \text { or } \\
\text { mean }[S D]\end{array}$ & $\begin{array}{l}\text { APR(n=31) } n(\%) \text { or } \\
\text { mean[SD] }\end{array}$ & $\mathbf{P}$ \\
\hline Operative time (minutes) & $261.8[96.6]$ & $319.2[112.4]$ & 0.021 \\
\hline Blood loss (mL) & $185.8[156.5]$ & $375.0[275.3]$ & 0.001 \\
\hline Number of harvested lymph nodes & $11.7[6.2]$ & $9.7[7.9]$ & 0.219 \\
\hline Anal or stoma exhaust (days) & $3.3[1.6]$ & $3.3[3.4]$ & 0.953 \\
\hline Time to soft diet (days) & $4.5[2.8]$ & $3.8[2.6]$ & 0.248 \\
\hline Postoperative hospital stay (days) & $15.5[8.5]$ & $20.4[14.0]$ & 0.087 \\
\hline $\begin{array}{l}\text { Postoperative thirty-day complications } \\
(+)\end{array}$ & $9(20.9)$ & $8(25.8)$ & 0.623 \\
\hline Anastomotic leakage & 3 & 0 & \\
\hline Wound infection & 2 & 2 & \\
\hline Hemorrhage & 1 & 4 & \\
\hline Urinary tract infection & 1 & 1 & \\
\hline lleus & 1 & 1 & \\
\hline Others & 1 & 0 & \\
\hline \multicolumn{4}{|l|}{ Clavien-Dindo score } \\
\hline 1 & $35(81.4)$ & $25(80.6)$ & 0.554 \\
\hline 2 & $3(6.9)$ & $4(12.9)$ & \\
\hline 3 & $5(11.6)$ & $2(6.4)$ & \\
\hline $\begin{array}{l}\text { Postoperative thirty-day } \\
\text { reoperation/readmission (+) }\end{array}$ & $1(2.3)$ & $1(3.2)$ & 0.814 \\
\hline Postoperative mortality & 1 & 0 & \\
\hline
\end{tabular}

\section{Pathological And Oncological Outcomes}

The average distance from the tumor to the anal verge in the ISR group was $4.5 \mathrm{~cm}$, while the APR group was $3.4 \mathrm{~cm}$, showing a statistically significant difference $(P=0.0001)$. No difference was noted in tumor size in the two groups. Two groups were similar in tumor differentiation $(P=0.337)$. Similarly, $p T$ stage 
and $\mathrm{pN}$ stage were comparable between the two groups. Each patient in two groups achieved R0 resection (Table 3).

Table 3

Pathological outcomes

\begin{tabular}{|c|c|c|c|}
\hline & $\begin{array}{l}\text { ISR }(n=43) n(\%) \text { or } \\
\text { mean[SD] }\end{array}$ & $\begin{array}{l}\text { APR }(n=31) n(\%) \text { or } \\
\text { mean[SD] }\end{array}$ & $\mathbf{P}$ \\
\hline Tumor height from AV (cm) & $4.5[0.7]$ & $3.4[1.1]$ & 0.0001 \\
\hline Tumor size $\left(\mathrm{cm}^{*} \mathrm{~cm}{ }^{*} \mathrm{~cm}\right)$ & $30.4[32.0]$ & $64.0[96.4]$ & 0.07 \\
\hline \multicolumn{4}{|l|}{ Tumor differentiation } \\
\hline Well & $3(7.0)$ & $3(9.7)$ & 0.337 \\
\hline Moderate & $39(90.7)$ & $25(80.5)$ & \\
\hline Poor & $1(2.3)$ & $3(9.7)$ & \\
\hline \multicolumn{4}{|l|}{ Pathological Depth } \\
\hline $\mathrm{T} 1 / \mathrm{T} 2$ & $27(62.8)$ & $13(41.9)$ & 0.076 \\
\hline T3/T4 & $16(37.2)$ & $18(58.1)$ & \\
\hline \multicolumn{4}{|l|}{$\begin{array}{l}\text { Pathological lymph node } \\
\text { metastasis }\end{array}$} \\
\hline Positive(+) & $13(30.2)$ & $8(25.8)$ & 0.677 \\
\hline Negative(-) & $30(69.8)$ & $23(74.2)$ & \\
\hline $\mathrm{RO}$ resection & $43(100 \%)$ & $31(100 \%)$ & \\
\hline $\mathrm{AV}=$ anal verge $\mathrm{APR}=\mathrm{abdo}$ & ineal resection; ISR & hincteric resection & \\
\hline
\end{tabular}

The median follow-up period was 26 months (interquartile range 13-54 months) and 39 months (interquartile range 18-58 months) in the ISR group and the APR group, respectively .

Three- and Five-year overall survival rates after ISR were $88 \%$ and $86 \%$, the $95 \%$ confidence interval for the mean survival time was $64.5-79.4$, and $77 \%$ and $57 \%$ (95\% $\mathrm{Cl}$ for the mean survival time $49.9-70.4)$ in the APR group, showing no statistically significant difference in patients' overall survival when comparing both groups ( $p=0.136$, see Fig. 2). The disease-free survival rates at 3 and 5 years were $79 \%$ and $72 \%$ in ISR, and $63 \%$ and $63 \%$ in the APR group, respectively. There was no significant difference in patients' disease-free survival between the two groups ( $p=0.303$, see Fig. 3 ). In terms of local recurrence, the 3year local recurrence rate was $5 \%$ in the ISR group and $19 \%$ in the APR group, with no significant difference between the two groups $(p=0.309$, see Fig. 4$)$.

\section{Functional Results}


We analyzed the functional data for 32 patients who received closure of the ileostomy after ISR. According to the low anterior resection syndrome score (LARS), of the 32 patients, 24 (75\%) scored between 0 and 20, indicating no low anterior resection syndrome, and 8 (25\%) scored between 21 and 29 , indicating mild low preresection syndrome. None scored above 30. The mean LARS score was 14.8. According to the Wexner score, twenty nine out of 32 patients had a score $<10(91 \%)$, representing good continence, 3 patients had a Wexner score $\geq 10(9 \%)$ and are regarded having a severely impaired continence. The mean Wexner continence score was 5.9. Overall, postoperative anal function in ISR patients was satisfactory.

\section{Discussion}

As laparoscopy and even robotics become more widely used and the understanding of gastrointestinal anatomy refined, more and more patients with low rectal cancer can achieve tumor resection and anal preservation through various of sphincter-saving surgery. However, for ultra-low rectal cancers near the anus, which are located too close to the anus and have too little pelvic space, especially in men, APR is still the standard procedure to achieve a negative resection margin, but the postoperative permanent to the pain of patients underwent colostomy and inconvenience is indelible, some patients even give up treatment because they could not accept postoperative colostomy. Thus, it has always been the research direction of gastrointestinal surgery to ensure the radical resection of tumor while preserving the patient's anus and maintaining the original gastrointestinal structure. ISR was first proposed by Professor Lyttle and Professor Parks in 1977 for the treatment of patients with inflammatory bowel disease requiring colon and rectal resection, and it was first used in 1994 to treat rectal cancer less than $5 \mathrm{~cm}$ from the anal margin. ISR dissects part or all of the internal sphincter by dissecting the sphincter space, and sutures the proximal colon and anal canal. It emphasizes the correct separation of the inner and outer sphincter. The range of internal anal sphincter resection based on tumor location is divided into complete ISR (complete resection of internal sphincter, resection at the sphincter groove), subtotal ISR (subtotal resection of internal sphincter, resection between dentate line and sphincter groove) and partial ISR (partial removal of the internal sphincter, removal at the dentate line) [11]. The proposal of ISR has brought hope to patients and the medical community, but also brought us a series of questions, such as the radical tumor problem and the anal function of patients after surgery.

This study compared a series of outcomes of ISR versus APR surgery for ultra-low rectal cancer patients in our center, including the short-term, oncological and functional outcomes. Our data showed that ISR may provide a feasible and safety alternative to APR based on the oncologic cure rate and defecation function.

In our study, ISR group showed shorter operative time and less blood loss than APR group. Moreover, the mean length of postoperative hospital stay was shorter in ISR group, although there were no statistical differences. In addition, 30-day complications and Clavien-Dindo score showed no statistical difference between the two groups, among them, anastomotic leakage was the main complication in the ISR group, while hemorrhage was the main complication in the APR group. Clinically, we consider that the main 
causes of anastomotic leakage including: (1) low anastomosis, difficult operation; (2) poor blood supply or excessive tension in the anastomosis; (3) more opportunities for pelvic and intestinal contamination. Because APR permanently closes the anus, the anastomotic leakage can be greatly reduced or avoided, and the postoperative complications mainly come from the large trauma caused by the surgery itself. Therefore, surgeons should fully understand the various complications, comprehensively evaluate patients before surgery, correctly grasp the surgical indications, make preventive stoma when appropriate, what's more, surgical skills and experience are also very important. These short outcomes demonstrated that ISR may provide a safe alternative to APR, with less surgical trauma and faster recovery.

In recent years, the survival results of ISR and APR have been reported in several studies. Rullier et al. found that the combination of preoperative radiotherapy with sphincter-saving procedures not only may facilitate good perioperative and functional outcomes, but is also safe from the oncological point of view [12]. Schiessel et al. studied 121 rectal cancer patients who underwent ISR. After 16 years of follow-up, they found that there were no statistically significant differences in postoperative survival and recurrence rate between patients with ISR compared with low anterior resection (LAR) and APR [13]. Shunsuke Tsukamoto et al. followed up 285 patients (112 ISR and 173 APR) by propensity score matching and found the similar oncologic outcomes for ISR and APR without preoperative chemoradiotherapy in patients with low rectal cancer [14]. In our study, the overall survival, disease-free survival and local recurrence rate were similar between the two groups, R0 resection achieved $100 \%$ in both the groups and the number of lymph nodes harvested showed no difference, indicating that oncological outcomes and surgical efficacy were similiar between ISR and APR. In addition, we found that postoperative local recurrence or metastasis in the ISR group mostly occurred in patients with poorly differentiated tumors, or with a small number of lymph node detection, suggesting that ISR surgery should be carefully selected for patients with poorly differentiated tumors with very low rectal cancer. For patients with insufficient lymph node detection, postoperative follow-up interval should be shortened and close observation should be conducted.

Although our study and another previous studies have confirmed the oncology safety of ISR, whether patients can obtain good stool control after surgery due to partial or complete loss of the internal sphincter is always a concern. Most scholars believe that preserving all internal sphincter and mucosa is the key to maintaining good stool control function after the surgery [15]. When the distance between the lower edge of the tumor and the anorectal ring is less than $2 \mathrm{~cm}$, most or even all internal sphincters need to be removed to ensure the radical treatment effect, and the patient will have severe defecation control dysfunction and even anal incontinence. To avoid this outcome, APR is often preferred. Due to the removal of part or all of the internal sphincter in ISR surgery, defecation control dysfunction will inevitably occur after a period of time, but the ability to control defecation can be recovered to varying degrees within 3 to 6 months after surgery. In our study, most of the patients after ISR presented with low anterior resection syndrome to varying degrees, but according to LARS score and Wexner score, the anal function of the vast majority of patients after ISR was satisfactory. Yamada et al. [16] also considered that postoperative anal function of ISR was generally satisfactory, and found no significant difference in postoperative defecation times between patients with partial internal sphincter resection and patients 
with subtotal resection, but anal function was relatively poor in patients with complete ISR. Therefore, it is of great significance to retain as much internal anal sphincter as possible to maintain good postoperative stool control. Motoi Koyama et al. [17] suggested that younger patients with T1 or T2 rectal cancers who require no preoperative therapy are ideal candidates for ISR. We believe that laparoscopy has significant implications for the safety and efficacy of oncology. With the help of the magnification effect of the laparoscope, the visual field can be exposed more clearly, the clearance and approach selection can be more accurate, and the perirectal tissue damage can be more effectively avoided. At the same time, the principle of total mesorectal excision should be strictly followed, and the peripheral incision edge should be kept smooth and intact, and the instinctual sense receptors located outside the intestinal wall should be preservated. This is of great significance for the recovery of postoperative anal function.

Currently, comprehensive treatment is emphasized for low and ultra-low rectal Cancer. According to the latest National Comprehensive Cancer Network (NCCN) guidelines, neoadjuvant radiotherapy and chemical therapy are recommended for $\mathrm{t} 3-4$ or patients with positive lymph node metastasis, which can reduce the tumor stage, reduce the positive rate of circumcircumential resection edge and improve the anal retention rate. However, many scholars believe that neoadjuvant chemoradiotherapy would increase the incidence of anastomotic fistula and impair postoperative anal function [18]. Ito etc. [19] had performed postoperative anal function evaluation in 96 cases of patients with the ISR, single factor analysis showed that excessive internal sphincter resection range and preoperative radiotherapy and chemical drug treatment is the adverse factors that affect the anus function, multi-factor analysis showed that only the preoperative radiation therapy and chemical therapy is the independent factors affect the anus function, has nothing to do with internal sphincter resection range. In order to avoid the adverse effects of neoadjuvant therapy on postoperative anal function and anastomotic healing, all patients did not receive preoperative radiotherapy or chemotherapy in this study.

The limitations of this study are retrospective and nonrandomized design of a single center and selection bias of patient. In addition, the sample size is also limited and the skill and experience of the surgeon may influence the outcome. Therefore, it is necessary to expand the sample size and carry out multicenter research.

\section{Conclusion}

In selected cases, ISR may provide a feasible and safe alternative to APR with good clinical, pathological, and oncological outcomes, besides, the anal functional outcomes after ISR are acceptable. In the future, efforts should be taken to limit the indications for APR. Moreover, larger prospective randomized studies and evaluation of more long-term functional results and patient quality of life are needed to confirm and update the conclusion.

\section{Declarations}

Acknowledgements

Page 10/16 
Jie Cao, Wanglin Li, Baifu Peng designed the study. Baifu Peng, Zixin Wu, ZiJian He carried out the collection, analysis, interpretation of data, and drafted the article. Guanwei Li searched the literature. All authors participated in revising the article and reached final approval of the version to be published.

The authors have no conflicts of interest or financial ties relevant to the present study. This work was supported by grants from Guangzhou Science Technology and Innovation Commission (201804010073).

Study performed institution: Department of Colorectal Surgery, Guangzhou First People's Hospital, School of Medicine, South China University of Technology

Conflicts of interest: The authors have no conflicts of interest related to this article to disclose.

Source of Funding: Guangzhou Science Technology and Innovation Commission (201804010073)

Ethics and consentᄆThe study was approved by the Guangzhou First People's Hospital ethics committee. All participants were informed of the study and signed a consent form to participate.

Consent for publication: The written informed consent for publication was obtained.

Availability of data and materials: All data generated or analysed during this study are included in this published article and its supplementary information files.

Author contributions: Jie Cao, Wanglin Li, Baifu Peng designed the study. Baifu Peng, Zixin Wu and ZiJian He carried out the collection, analysis, interpretation of data, and drafted the article. Guanwei Li searched the literature. All authors participated in revising the article and reached final approval of the version to be published.

\section{References}

1. Battersby NJ, How P, Moran B, et al. Prospective Validation of a Low Rectal Cancer Magnetic Resonance Imaging Staging System and Development of a Local Recurrence Risk Stratification Model. Ann Surg. 2016;263(4):751-60.

2. Bray F, Ferlay J, Soerjomataram I, et al. Global cancer statistics 2018: GLOBOCAN estimates of incidence and mortality worldwide for 36 cancers in 185 countries. CA Cancer J Clin. 2018;68(6):394-424.

3. Perry WB, Connaughton JC. Abdominoperineal resection: how is it done and what are the results? Clin Colon Rectal Surg. 2007;20(3):213-20.

4. Rullier E, Laurent C, Bretagnol Fdr, et al. Sphincter-Saving Resection for All Rectal Carcinomas. Ann Surg. 2005;241(3):465-9.

5. Kim IY, Kim BR, Kim YW. Outcomes of laparoscopic and open surgery for colorectal cancer in the emergency setting. In vivo (Athens, Greece). 2015; 29(2):295-300. 
6. Huang M-Y, Lee H-H, Tsai H-L, et al. Comparison of efficacy and safety of preoperative Chemoradiotherapy in locally advanced upper and middle/lower rectal cancer. Radiation Oncology. 2018; 13(1).

7. Wu X, Lin G, Qiu H, et al. Loop ostomy following laparoscopic low anterior resection for rectal cancer after neoadjuvant chemoradiotherapy. European Journal of Medical Research. 2018; 23(1).

8. Dindo D, Demartines N, Clavien P-A. Classification of Surgical Complications Annals of Surgery. 2004;240(2):205-13.

9. Emmertsen KJ, Laurberg S. Low Anterior Resection Syndrome Score. Ann Surg. 2012;255(5):922-8.

10. Jore JM, Wexner SD. Etiology and management of fecal incontinence. Dis Colon Rectum. 1993;36(1):77-97.

11. Akagi Y, Kinugasa T, Shirouzu K. Intersphincteric resection for very low rectal cancer: a systematic review. Surg Today. 2012;43(8):838-47.

12. Rullier $E$, Laurent $C$, Zerbib $F$, et al. Conservative treatment of adenocarcinomas of the anorectal junction by preoperative radiotherapy and intersphincteral resection. Ann Chir. 2000;125:618-24.

13. Schiessel R, Novi G, Holzer B, et al. Technique and long-term results of intersphincteric resection for low rectal cancer. Dis Colon Rectum. 2005;48(10):1858-65. discussion 1865 - 1857.

14. Tsukamoto S, Miyake M, Shida D, et al. Intersphincteric Resection Has Similar Long-term Oncologic Outcomes Compared With Abdominoperineal Resection for Low Rectal Cancer Without Preoperative Therapy: Results of Propensity Score Analyses. Dis Colon Rectum. 2018;61(9):1035-42.

15. Saito N, Moriya Y, Shirouzu K, et al. Intersphincteric Resection in Patients with Very Low Rectal Cancer. Diseases of the Colon Rectum. 2006;49:13-22.

16. Yamada K, Ogata S, Saiki Y, et al. Functional results of intersphincteric resection for low rectal cancer. Br J Surg. 2007;94(10):1272-7.

17. Koyama M, Murata A, Sakamoto $Y$, et al. Long-term clinical and functional results of intersphincteric resection for lower rectal cancer. Ann Surg Oncol. 2014;21(Suppl 3):422-8.

18. Klose J, Tarantino I, Kulu Y, et al. Sphincter-Preserving Surgery for Low Rectal Cancer: Do We Overshoot the Mark? J Gastrointest Surg. 2017;21(5):885-91.

19. Ito M, Saito N, Sugito M, et al. Analysis of Clinical Factors Associated with Anal Function after Intersphincteric Resection for Very Low Rectal Cancer. Diseases of the Colon Rectum. 2009;52(1):64-70.

\section{Figures}


FIG. 1. Patients selection.

1436 patients affected by CRC

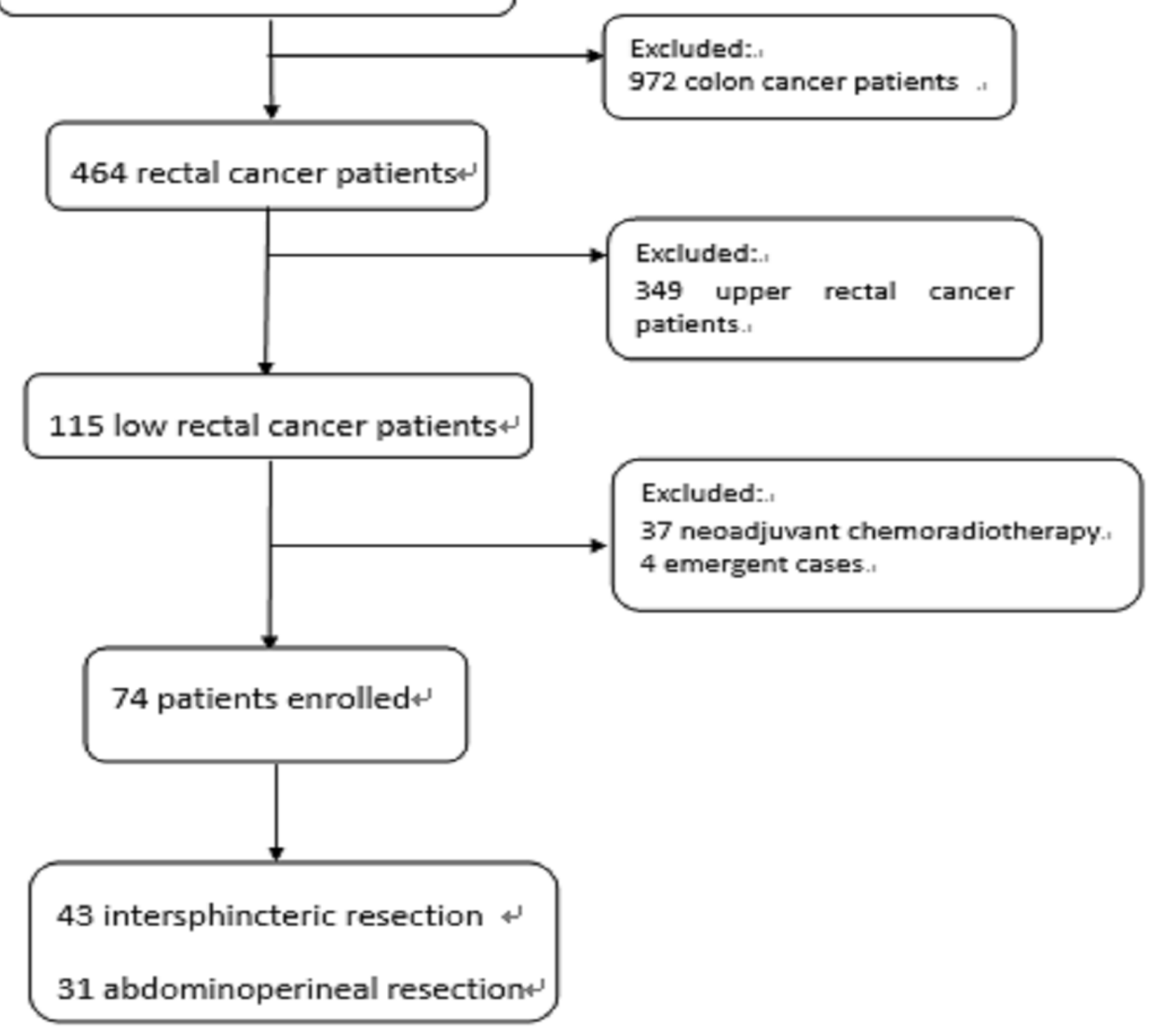

Figure 1

Patients selection 


\section{Overall Survival}

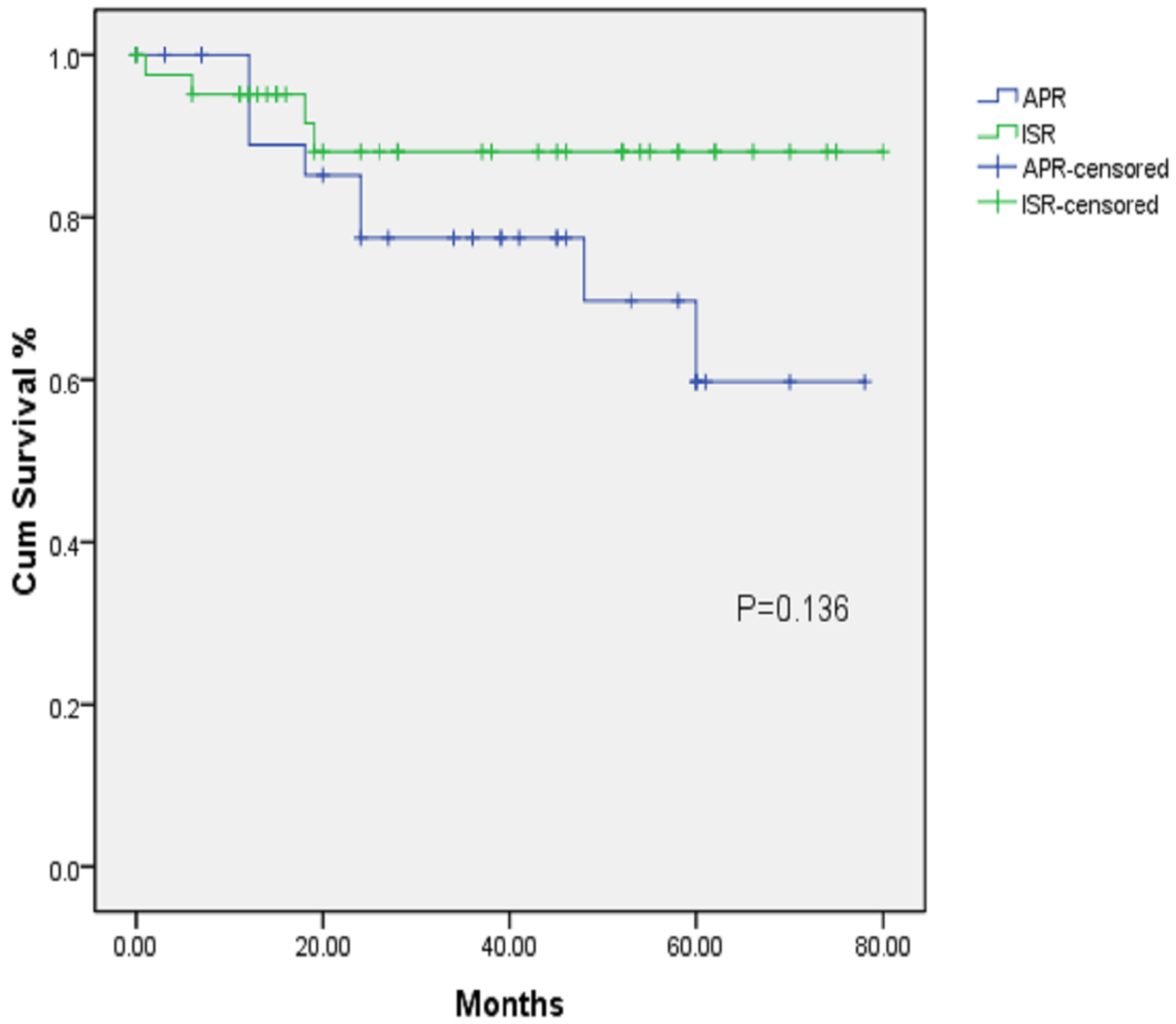

Figure 2

Overall survival between the two groups ISR= Intersphincteric Resection. APR=Abdominoperineal Resection 
Disease-free Survival

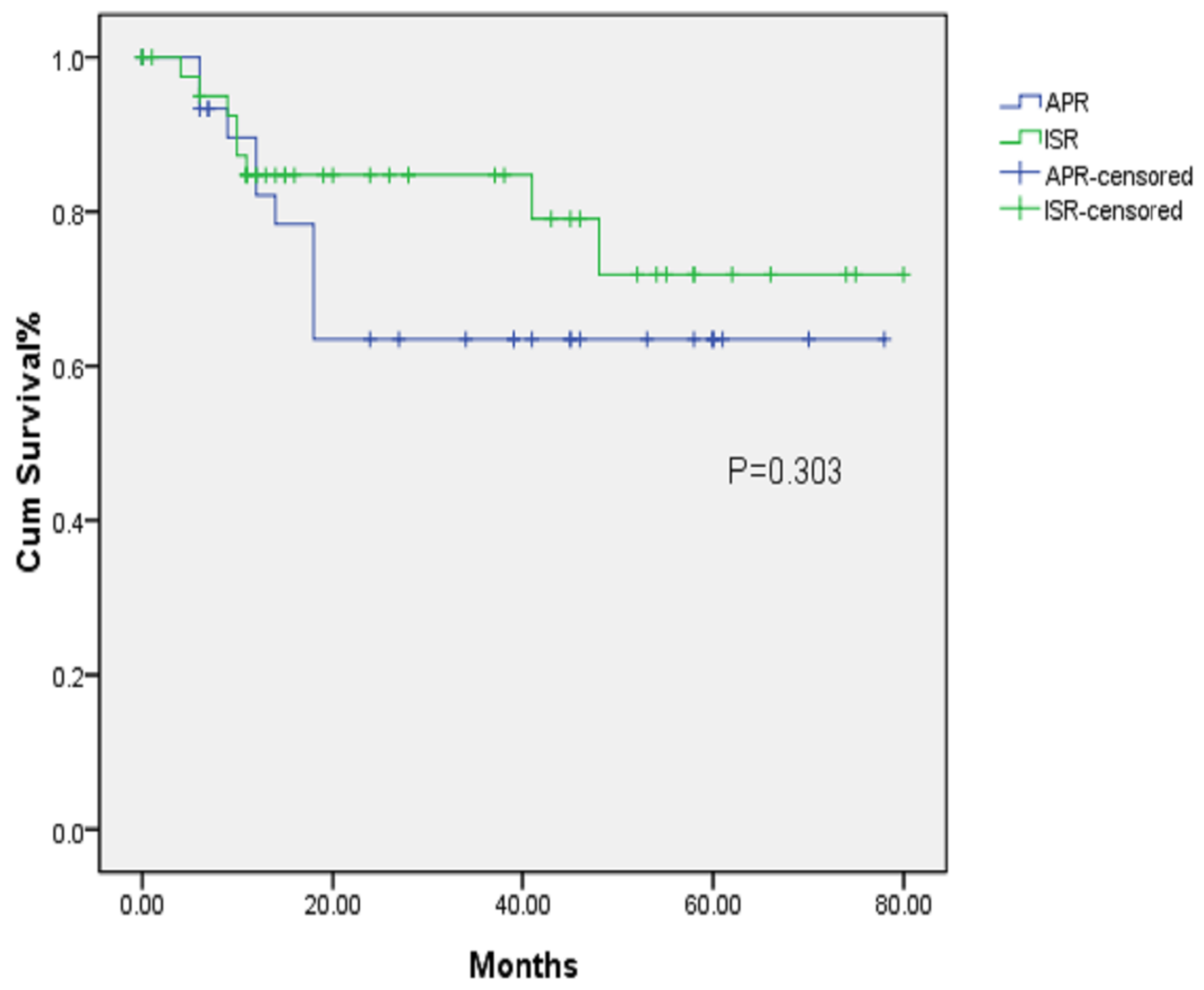

Figure 3

Disease-free survival between the two groups ISR= Intersphincteric Resection. APR=Abdominoperineal Resection 


\section{Cumulative local recurrence}

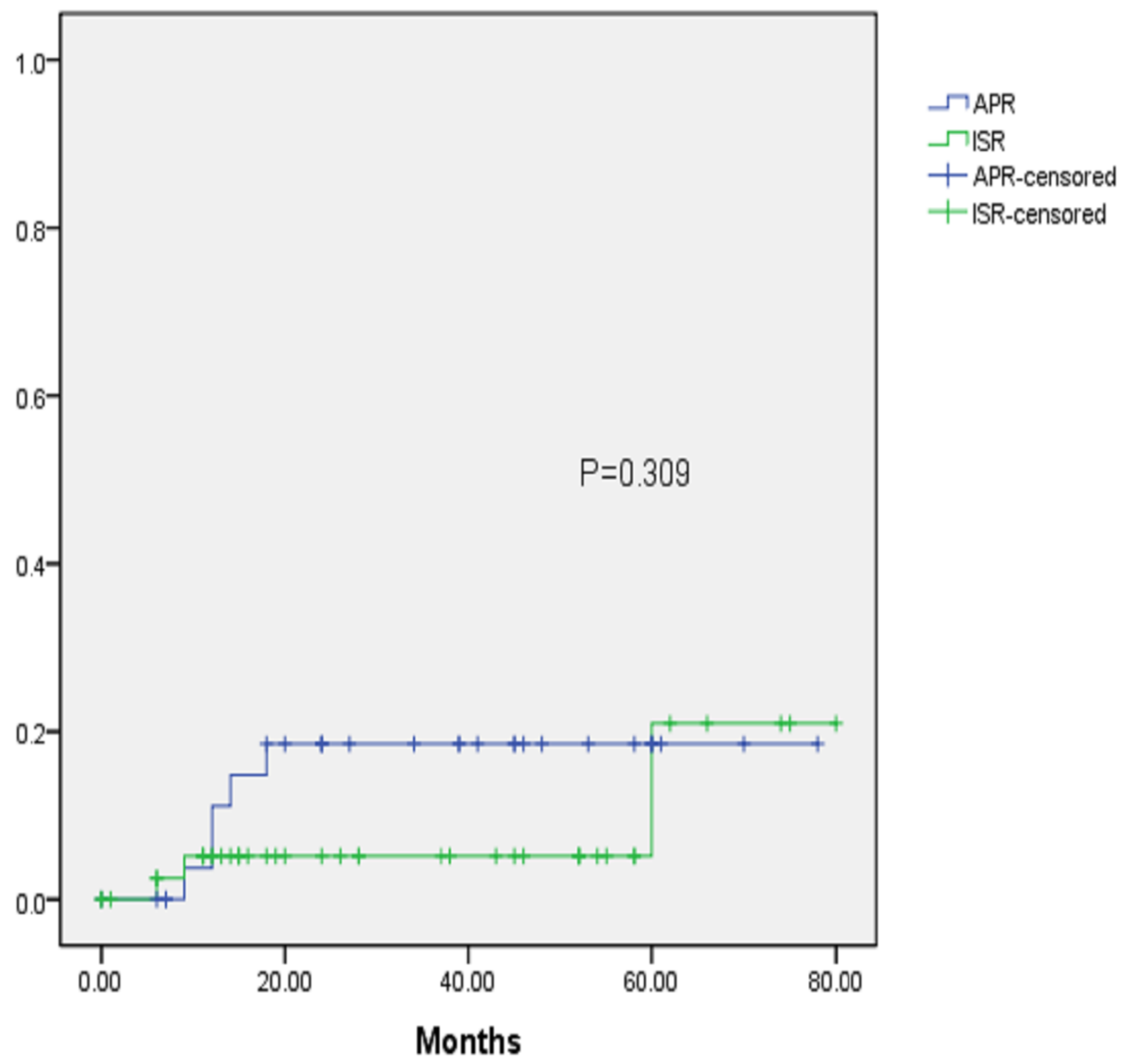

\section{Figure 4}

Local recurrence rates between the two groups ISR= Intersphincteric Resection. APR=Abdominoperineal Resection 